\title{
Reversible switching of polymeric gel structure and property by solvent exchange
}

\author{
Liju $\mathrm{Xu}^{1}$ and Dong Qiu ${ }^{1,2 *}$
}

The distinctive attributes of the dynamic polymeric gels, such as configurable mechanical behavior [1-6], external stimuliresponsiveness [7-13] and self-healing ability [14-17], underlie their extensive applications in materials science, ranging from tissue-adaptive biomaterials [18-20] through high-efficient energy materials [21-23] to intelligent information materials [24-26]. For instance, stiffness-changing trait (e.g., rigid-to-soft transition) is especially desired in medical devices, since the rigid state allows for the easy insertion, while the soft state favors biocompatibility with surrounding tissues [19,27]. Therefore, tremendous efforts have been devoted to imparting dynamic features to polymer networks through designing unique chemical compositions and structural motifs, which are capable of transforming the chemical structure, topological structure or conformational structure of the cross-links [1,27-29]. However, those existing approaches either require dedicated and sophisticated chemical design or suffer from narrow operational state and poor reversibility $[1,19,28,29]$. Overall, there remains a significant need for effective strategies to continuously and reversibly regulate the structures and properties of polymeric networks.

For the design of polymeric gel materials, the solvent composition and content are general parameters that are crucial in determining the properties and functions of the swollen polymeric networks [30-33]. Recently we reported a class of robust and nonswellable hydrogels (named as exogels) by regulation of noncovalent interactions via solvent exchange [32]. The key to this approach is that the intra- and interpolymer interactions are transiently shielded to maintain extended conformations and percolating architectures, and then recover to establish a stiff and tough network through displacing a good solvent (e.g., dimethyl sulfoxide, DMSO) with a poor one (i.e., water). Simultaneously, various organic solvents, such as glycol, glycerol and DMSO, offer the possibility of fabricating hybrid organohydrogels with an improved working temperature range and long-term application capability [34-36]. Therefore, it inspires us to employ various solvent pairs to switch the polymeric network's structures and performances via solvent exchange.

Herein, utilizing the competition between the interaction of polymer-polymer and interplay of polymer-solvent, we proposed solvent switching as a strategy to develop the dynamic exogel in which the network architecture and multiple aspects of properties were regulated coherently. A loose polymeric network was achieved when polymer self-interactions were switched off by binding with a good solvent. Upon switching to another relatively poor solvent, the interactions among the polymers were restored and they assembled into a dense network. Therefore, the aggregation of the polymer chains and the structures of polymer networks can be altered by solvent switching to dynamically and reversibly modulate the mechanical properties in the dynamic exogel system. Meanwhile, the non-drying and anti-freezing feature of the terminal solvent endowed the dynamic exogel with improved and broadened working temperature range, paving the way toward versatile application scenarios.

By introducing the competition of configurable interactions established between polymer and solvent and the ones formed inside each, the dynamic and stimuli-responsive exogel system with tunable mechanical properties and wide temperature allowances was designed and constructed via our previously proposed solvent-exchange strategy as illustrated in Fig. 1a. Concretely, owing to its strong hydrogen bond accepting capability, DMSO as a good solvent was selected as the initial solvent to dissolve polymers such as polyvinyl alcohol (PVA), where polymer-solvent hydrogen bonds were energetically preferred over polymer-polymer hydrogen bonds, thus resulting in a loose network pattern. Accompanied with the solventexchange operation to introduce a regulator (e.g., water) that allowed for competitive hydrogen bonding, the extent of the PVA self-hydrogen bonding can be dynamically altered to switch the architecture of the network. With the establishment of stable hydrogen bonding between DMSO and water that restricted their hydrogen-bonding capacity with the PVA chains (i.e., the DMSO/water binary solvent as a poor solvent), PVA chains would associate with each other to restore extensive interpolymer hydrogen bonds, leading to a densely packed network structure. Furthermore, the cross-linking density of the polymer network became moderate when DMSO was totally displaced with water, because the solvency of water for PVA was between that of DMSO and DMSO/water binary solvent. Consequently, the mechanical performances of the exogel can be selectively and reversibly modulated by tuning the polymeric network structure through the addition or removal of the solvent-regulator. Simultaneously, the freezing point and vapor pressure of the DMSO/water binary solvent system can also be altered by changing the volume ratio due to intermolecular interactions between DMSO and water, thus tuning the temperature tolerance of the dynamic exogels.

\footnotetext{
${ }^{1}$ Beijing National Laboratory for Molecular Sciences, Laboratory of Polymer Physics and Chemistry, CAS Research/Education Center for Excellence in Molecular Sciences, Institute of Chemistry, Chinese Academy of Sciences, Beijing 100190, China

${ }^{2}$ University of Chinese Academy of Sciences, Beijing 100049, China

* Corresponding author (email: dqiu@iccas.ac.cn)
} 

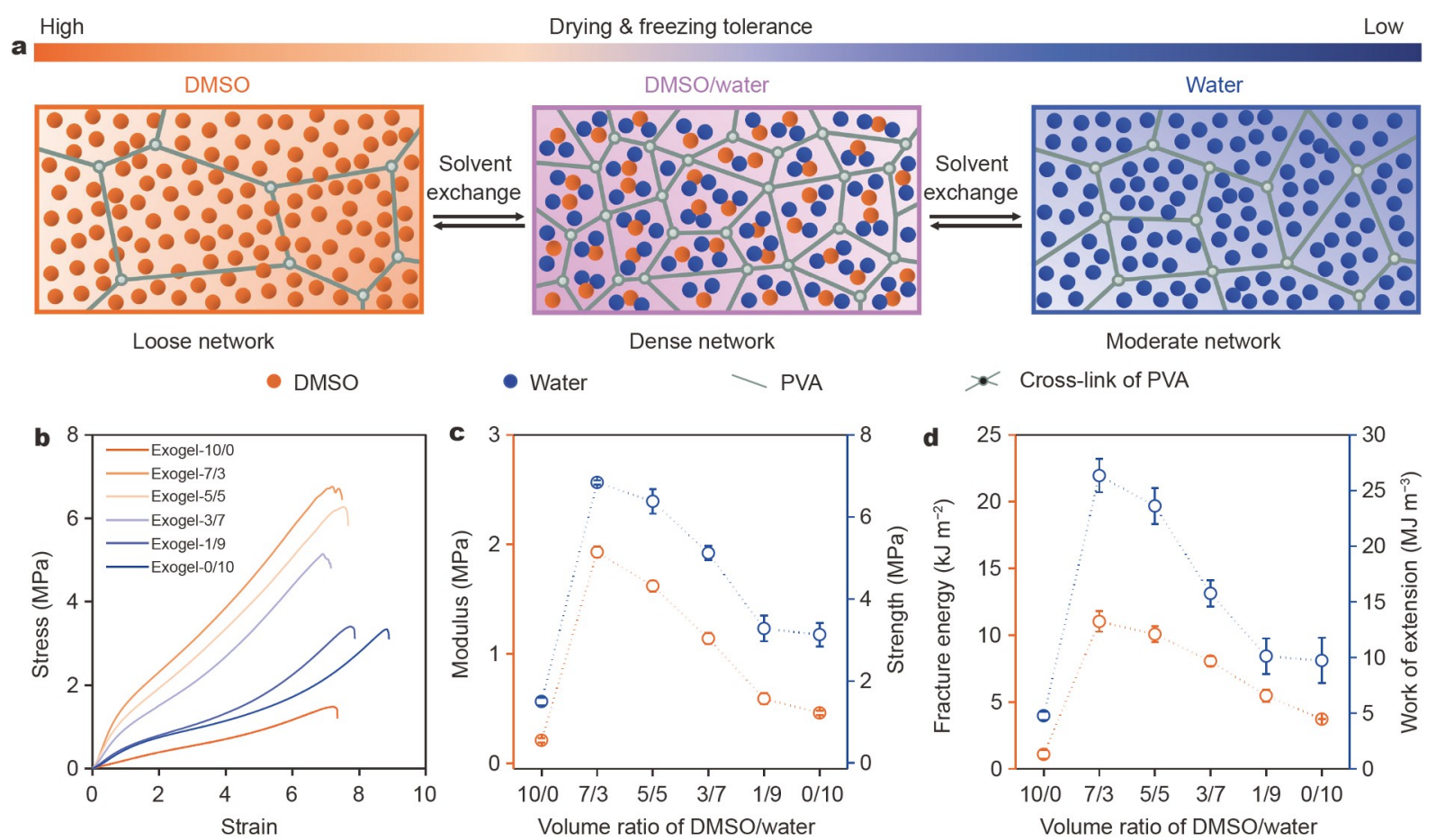

Figure 1 Reversible switching of the polymeric network structures and properties by solvent exchange. (a) Conceptual scheme of regulating the interpolymeric interactions to modulate the network structure by solvent exchange. (b) Tensile stress-strain curves of the dynamic PVA exogels by coherently varying the volume ratio of DMSO/water in the final solvents, ranging from 10/0 to $0 / 10$. Switchable mechanical properties of the dynamic exogel, including (c) tensile modulus and strength and (d) fracture energy and work of extension. The error bars represent standard deviation; sample size $n=3-5$.

The water volume fraction of the final solvent in the exogel can be readily and precisely adjusted from $0 \%$ to $100 \%$ by solvent exchange in a series of DMSO/water binary solvents, where the volume ratio of $\mathrm{DMSO} /$ water ranged from $10 / 0$ to $0 / 10$. To facilitate further discussion, we denoted a variety of exogels using the abbreviation exogel- $x / y$, where $x / y$ represented the volume ratio of DMSO and water. As demonstrated in Fig. 1b, we found that the dynamic PVA exogel realized a significant transformation of mechanical behaviors between softness and rigidity by controlling the water content. Quantitatively, the tensile modulus of the PVA exogel enhanced from $0.21 \pm 0.02$ to $1.93 \pm 0.05 \mathrm{MPa}$ (i.e., a modulus contrast by a factor of $\sim 10$ ) upon varying the volume ratio of DMSO/water from $10 / 0$ to $7 / 3$, and then exhibited a decreasing trend from $1.93 \pm 0.05$ to $0.46 \pm$ $0.02 \mathrm{MPa}$ corresponding to the change in volume ratio of $\mathrm{DMSO} /$ water from $7 / 3$ to $0 / 10$ (Fig. 1c). Similarly, the tensile strength and toughness also displayed the same trend with the change of volume fraction of water in the final solvents. As displayed in Fig. 1d and Fig. S1, the fracture energy of the exogel-7/3 was significantly improved to $11.03 \pm 0.76 \mathrm{~kJ} \mathrm{~m}^{-2}$, which was $\sim 10$ and $\sim 3$ times that of the exogel-10/0 (1.08 \pm $\left.0.33 \mathrm{~kJ} \mathrm{~m}^{-2}\right)$ and exogel-0/10 $\left(3.72 \pm 0.02 \mathrm{~kJ} \mathrm{~m}^{-2}\right)$. In other words, the PVA exogel demonstrated the most superb mechanical properties when the volume ratio of $\mathrm{DMSO} /$ water reached $7 / 3$ (i.e., molar ratio close to $1 / 2$ ), because the most extensive interpolymer hydrogen bonding was restored due to the rather stable state for DMSO-water hydrogen bonding in the pattern of 1DMSO-2water aggregates (i.e., one DMSO molecule bound with two molecules of water) $[35,36]$. Moreover, the reconfigurable hydrogen bonding also empowered the dynamic PVA exogels with excellent self-recovery and anti-fatigue capa- city (Fig. S2). We applied the area ratio of the second hysteresis loop to the first as the recovery efficiency and noticed that the recovery efficiency of the PVA exogel-7/3 can exceed $75 \%$ after a relatively short relaxing time ( $\sim 60 \mathrm{~min})$. Besides, there was no substantial plastic deformation and strength reduction after ten loading-unloading cycles. It was worth mentioning that the exogel exhibiting large mechanical changes had approximately the same solid content and concentration of polymer (Fig. S3), which further confirmed that the polymer network structure played a critical role in determining the mechanical properties.

Consistent with the proposed mechanism, the switching of mechanical states on the PVA exogel was reversible. As shown in Fig. S4, by cyclically regulating the volume ratio of DMSO/water in the final solvent between $7 / 3$ and $10 / 0$, both tensile modulus and strength can be cyclically and reversibly regulated between exogel-7/3 ( 1.8 and $\sim 6.0 \mathrm{MPa})$ and exogel-0/10 ( 0.4 and $\sim 3.0 \mathrm{MPa}$ ), implying the outstanding and stable reversibility of mechanical behaviors. Meaningfully, the solvent-exchange protocol was straightforward and universal to continuously and reversibly tune the mechanical properties of the exogels over a broad range for versatile applications, which can be readily achieved by either varying the components of the solvent or the extent of solvent displaced.

The attenuated total reflection Fourier transform infrared (ATR-FTIR) spectroscopy was used to investigate the competitive hydrogen bonding in the system. The full ATR-FTIR spectrum was displayed in Fig. S5, and local ATR-FTIR spectra showing the stretching and bending vibration alteration for $\mathrm{O}-\mathrm{H}$ and the stretching vibration alteration for $\mathrm{S}=\mathrm{O}$ were summarized in Fig. $2 \mathrm{a}-\mathrm{c}$. As water was introduced as the regulator, the $\mathrm{S}=\mathrm{O}$ stretches moved to lower wavenumbers with increasing volume 

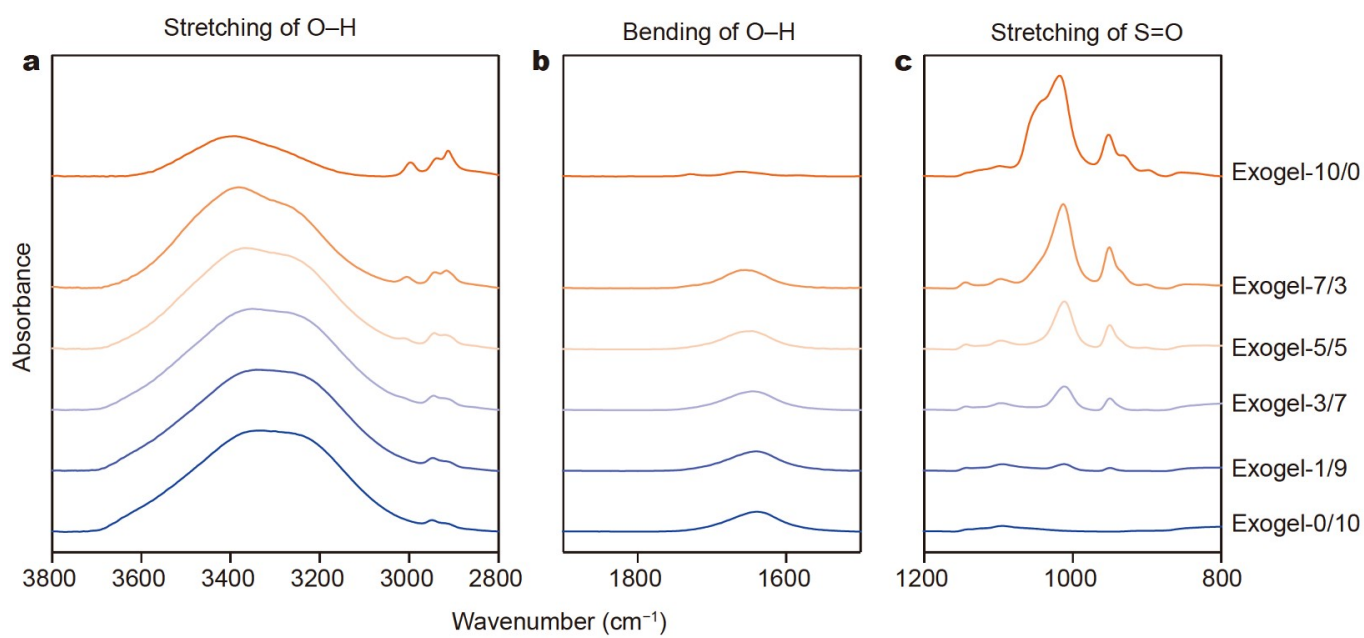

d
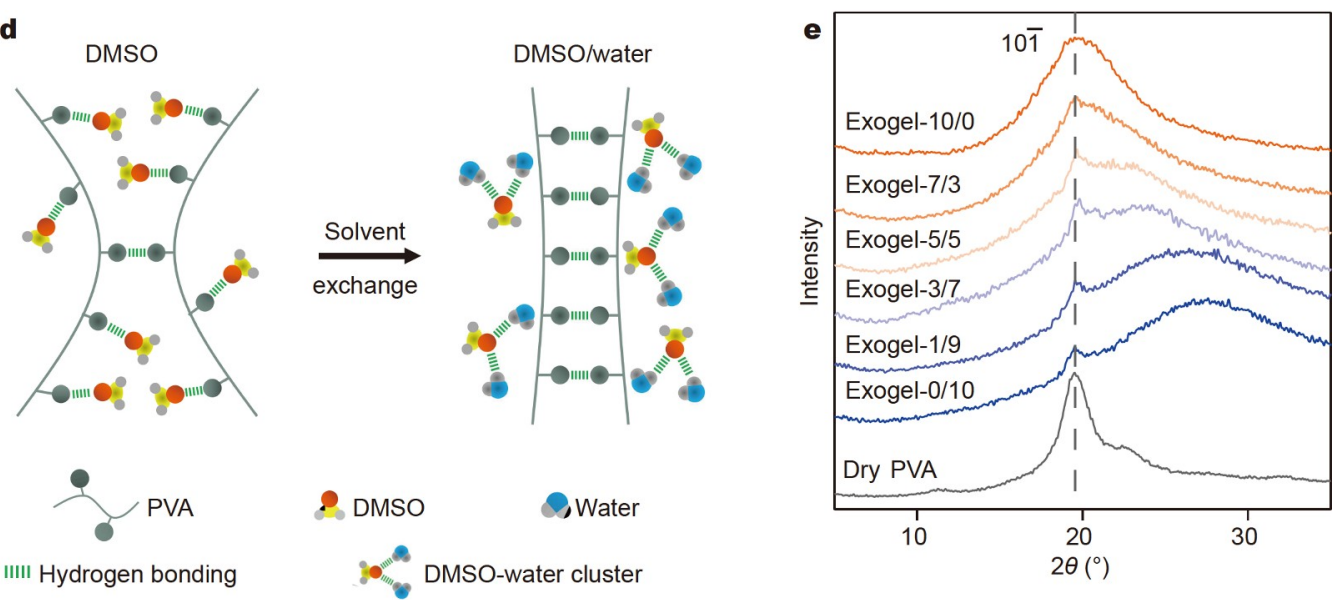

Figure 2 Characterization of competitive hydrogen bonding and crystalline domains in the dynamic PVA exogel system. (a-c) ATR-FTIR spectra of PVA exogels prepared by exchanging DMSO with DMSO/water mixture with different volume fractions of DMSO. Local ATR-FTIR spectra showing the (a) stretching and (b) bending vibration alterations for $\mathrm{O}-\mathrm{H}$ and (c) the stretching vibration alteration for $\mathrm{S}=\mathrm{O}$. (d) Schematics of the competition between the hydrogen bonding of polymer-polymer and that of polymer-solvent, the hydrogen bonding between PVA molecules was suppressed in DMSO owing to strong hydrogen bond accepting capability of DMSO, while restored in the DMSO/water binary solvent due to the weak solvency induced by rather stable hydrogen bonding of DMSO-water cluster. (e) XRD profiles of PVA exogels using various DMSO/water binary solvents as the final solvents.

fraction of water, implying that the hydrogen bonding network where DMSO and water molecules participated was built and gradually became stronger. In addition, both of the stretching and bending bands of $\mathrm{O}-\mathrm{H}$ showed an apparent blue-shift in the mixture of $\mathrm{DMSO} /$ water. The possible explanation for the above results was that hydrogen bonds among water molecules were weakened in the DMSO/water mixtures, because hydrogen bonds between DMSO and water were more energetically stable than those in pure water. In short, strong hydrogen bonds can be preferentially formed between DMSO and water molecules, giving rise to the weak hydrogen bonding capacity with the PVA chains, which thus maximized the hydrogen bonding interactions among PVA chains and therewith enhanced the bulk mechanical property of the exogel (Fig. 2d).

Generally, the crystalline domains were well known as the cross-links in the PVA physical network, which had a profound effect to their mechanical properties [32]. X-ray diffraction (XRD) was conducted to confirm that the mechanical changes observed above were due to changes in the crystalline domains (i.e., cross-linked network structures) within the exogel. As shown in Fig. 2e, the PVA exogel-10/0 had no apparent crys- talline peaks, indicating there were few crystalline aggregates in the exogel. By contrast, upon the introduction of water, the PVA exogel showed an obvious diffraction peak at $2 \theta=19.7^{\circ}$, which corresponded to the typical reflection plane of $(10 \overline{1})$ in semicrystalline PVA. This was because the $\mathrm{DMSO} /$ water binary solvent and water were not as good solvent as DMSO and the poor solvation of PVA chains facilitated the crystallization of PVA. Taken together, the crystallinity versus solvents trend of the dynamic PVA exogel system further supported and was matched well with the correlation between the mechanical performances and final solvent conditions.

In addition to the reversible switching of mechanical properties, the dynamic exogel also exhibited the unique wide temperature tolerance, which is important for sustaining functions and long-term stability in practical applications, especially under extreme conditions. On account of the establishment of hydrogen-bonding-stabilized DMSO-water molecular clusters, the $\mathrm{DMSO} /$ water binary solvent showed reduced vapor pressure and endowed the PVA exogel with prominent non-drying performances. As shown in Fig. 3a and Fig. S6, the weight retention of the fabricated PVA gels was heavily dependent on the volume 

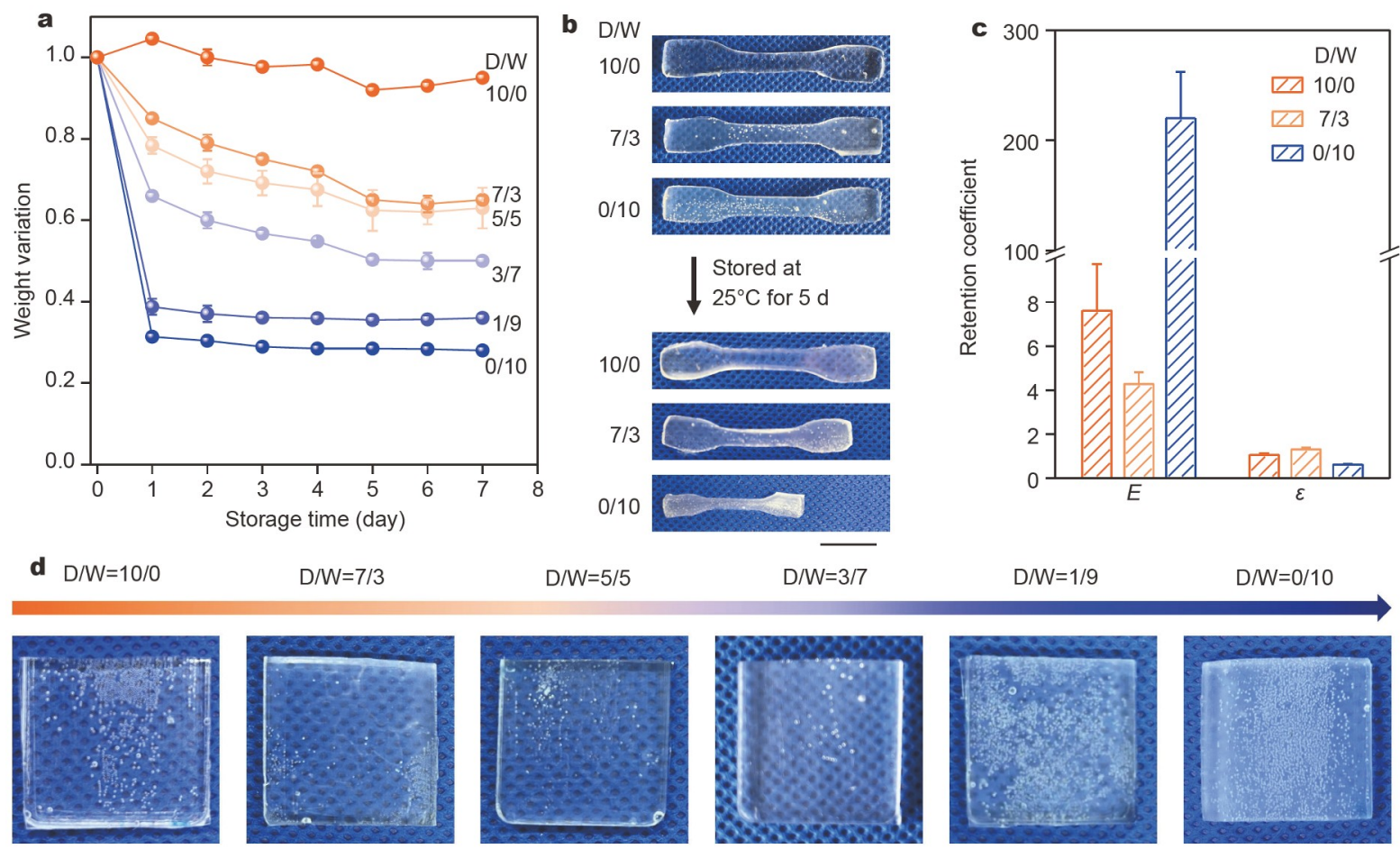

Stored at $-25^{\circ} \mathrm{C}$ for $48 \mathrm{~h}$
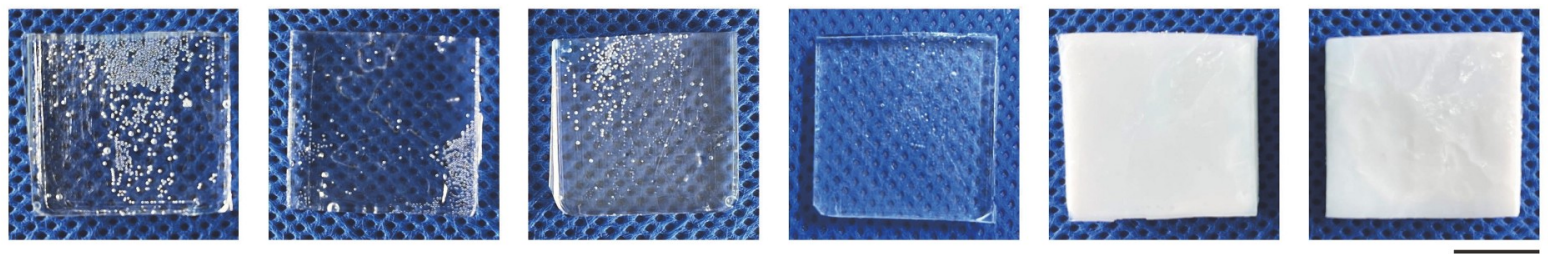

Figure 3 Non-drying and anti-freezing behaviors. (a) Weight retention of the PVA exogels with varying volume fraction of DMSO in the terminal solvents upon storing at $25^{\circ} \mathrm{C}$ and $50 \%$ humidity. (b) Optical photos and (c) retention coefficient of elastic modulus $(E)$ and fracture strain $(\varepsilon)$ of the PVA exogel-10/0, exogel-7/3 and exogel-0/10 (i.e., DMSO-based organogel, DMSO/water-based organohydrogel and water-based hydrogel) after storage at $25^{\circ} \mathrm{C}$ and $50 \%$ humidity for 5 days. (d) Optical photos of the PVA exogels with varying volume fraction of DMSO in the terminal solvents after storage at $-25^{\circ} \mathrm{C}$ for $48 \mathrm{~h}$. The PVA hydrogel turned into an ice-like solid state, while the PVA organohydrogels and organogel still remained transparent, implying the excellent freezing tolerance. Scale bar: $10 \mathrm{~mm}(\mathrm{~b}, \mathrm{~d})$. To facilitate display, the ratio of DMSO/water was denoted using the abbreviation D/W. The error bars represent standard deviation; sample size $n=3$.

ratio of DMSO to water in the terminal solvent. Unsurprisingly, by totally displacing DMSO with water, the PVA exogel-0/10 (i.e., hydrogel) shrunk significantly with a mass loss of $\sim 72 \%$ close to its water content after 5 days $\left(25^{\circ} \mathrm{C}, 50 \%\right.$ humidity) (Fig. 3b). In contrast, the PVA exogel with the DMSO/water binary solvent as the final dispersing medium, namely PVA organohydrogel, demonstrated much better solvent retention. Under the same condition, the weight retention enhanced from $35 \% \pm 1 \%$ to $65 \% \pm 1 \%$ when increasing the volume fraction of DMSO from $10 \%$ to $70 \%$, implying the effective anti-drying property. For the PVA exogel-10/0 without water introduced (i.e., organogel), an increase in weight was observed, which can be ascribed to the hygroscopic nature of the DMSO (i.e., water molecules can be captured by the DMSO-based PVA organogel from the moisture). Consequently, the PVA organogel and organohydrogel still remained highly stretchable and tough after storage for 5 days, while the dehydrated PVA hydrogel became rigid and obviously yield comparable to the water-less plastics (Fig. 3c and Fig. S7). Quantitatively, the tensile modulus of the dehydrated PVA exogel-0/10 was significantly increased to
101.12 $\pm 19.20 \mathrm{MPa}$ after storage for 5 days, accounting for $\sim 220$ times higher than the as-made PVA exogel-0/10. On the contrary, both of the PVA exogel-10/0 and exogel-7/3 still remained relatively low modulus of $1.63 \pm 0.45$ and $8.27 \pm 1.00 \mathrm{MPa}$, respectively, revealing their excellent non-drying properties.

Apart from the non-drying property, the DMSO/water mixture also endued the PVA exogel system with outstanding antifreezing performances by interrupting the hydrogen bonding network of pure water to restrain ice formation at sub-zero temperatures. As shown in Fig. 3d, all the PVA exogels were transparent at $25^{\circ} \mathrm{C}$, but the PVA exogel-0/10 and exogel-1/9 turned into white opaque color when frozen at $-25^{\circ} \mathrm{C}$ for $48 \mathrm{~h}$, due to the formation of ice crystals within the exogel. In contrast, the other PVA organohydrogels and organogel still maintained transparent and flexible under the same condition. Over a wide temperature range, the integration of the impressive drying and freezing tolerant behaviors on the stimuli-responsive PVA exogel can broaden the scope of their applications as flexible electronics, medical devices and electronic skin in extreme conditions. 
Capitalizing on the competition between the dynamic interactions of polymer-polymer and those of polymer-solvent, we applied a solvent-exchange strategy to reversibly regulate the network structures of the exogel. As such, the mechanical properties of the dynamic exogel can selectively and reversibly be switched between a soft state and a rigid state through arranging its network architecture between a loose mode and a compact mode in response to specific trigger of solvent. Moreover, the combination of drying and freezing tolerances was also realized on the mechanically switchable PVA exogel because of robust hydrogen bonds formed between DMSO and water molecules, which prevented the water from evaporating and icing. We anticipated that the strategy reported in this work can shed light on the tailoring of structural and physical properties of polymeric networks on demand towards versatile applications in a broad scope of environments, such as mechanically adaptive medical devices and wearable electronics exposed to arid and low-temperature environments.

\section{Received 1 September 2021; accepted 9 October 2021; published online 12 November 2021}

1 Gu Y, Alt EA, Wang $\mathrm{H}$, et al. Photoswitching topology in polymer networks with metal-organic cages as crosslinks. Nature, 2018, 560: 6569

2 Yang FK, Cholewinski A, Yu L, et al. A hybrid material that reversibly switches between two stable solid states. Nat Mater, 2019, 18: 874-882

$3 \mathrm{Xu} \mathrm{L}$, Wang $\mathrm{C}$, Cui $\mathrm{Y}$, et al. Conjoined-network rendered stiff and tough hydrogels from biogenic molecules. Sci Adv, 2019, 5: eaau3442

4 Nonoyama T, Lee YW, Ota K, et al. Instant thermal switching from soft hydrogel to rigid plastics inspired by thermophile proteins. Adv Mater, 2020, 32: 1905878

5 Ming Z, Pang Y, Liu J. Switching between elasticity and plasticity by network strength competition. Adv Mater, 2020, 32: 1906870

6 Zhu CN, Li CY, Wang H, et al. Reconstructable gradient structures and reprogrammable 3D deformations of hydrogels with coumarin units as the photolabile crosslinks. Adv Mater, 2021, 33: 2008057

7 Wang ZJ, Zhu CN, Hong W, et al. Cooperative deformations of periodically patterned hydrogels. Sci Adv, 2017, 3: e1700348

8 Zhou Y, Duque CM, Santangelo CD, et al. Biasing buckling direction in shape-programmable hydrogel sheets with through-thickness gradients. Adv Funct Mater, 2019, 29: 1905273

9 Fan W, Shan C, Guo H, et al. Dual-gradient enabled ultrafast biomimetic snapping of hydrogel materials. Sci Adv, 2019, 5: eaav7174

10 Kim H, Kang JH, Zhou Y, et al. Light-driven shape morphing, assembly, and motion of nanocomposite gel surfers. Adv Mater, 2019, 31: 1900932

11 Ji X, Yao Y, Li J, et al. A supramolecular cross-linked conjugated polymer network for multiple fluorescent sensing. J Am Chem Soc, 2013, 135: 74-77

12 Hou Y, Deng X, Xie C. Biomaterial surface modification for underwater adhesion. Smart Mater Med, 2020, 1: 77-91

13 Peng T, Dang-i AY, Liu J, et al. [2+2] Photocycloaddition reaction regulated the stability and morphology of hydrogels. Adv Fiber Mater, 2019, 1: 241-247

14 Zhang M, Xu D, Yan X, et al. Self-healing supramolecular gels formed by crown ether based host-guest interactions. Angew Chem Int Ed, 2012, 51: 7011-7015

15 Liu J, Tan CSY, Yu Z, et al. Tough supramolecular polymer networks with extreme stretchability and fast room-temperature self-healing. Adv Mater, 2017, 29: 1605325

16 Jiang C, Zhang L, Yang Q, et al. Self-healing polyurethane-elastomer with mechanical tunability for multiple biomedical applications in vivo. Nat Commun, 2021, 12: 4395

17 Zuo H, Liu Z, Zhang L, et al. Self-healing materials enable free-standing seamless large-scale 3D printing. Sci China Mater, 2021, 64: 1791-1800
18 Yuk H, Varela CE, Nabzdyk CS, et al. Dry double-sided tape for adhesion of wet tissues and devices. Nature, 2019, 575: 169-174

19 Zhang D, Dashtimoghadam E, Fahimipour F, et al. Tissue-adaptive materials with independently regulated modulus and transition temperature. Adv Mater, 2020, 32: 2005314

20 Peng X, Xia X, Xu X, et al. Ultrafast self-gelling powder mediates robust wet adhesion to promote healing of gastrointestinal perforations. Sci Adv, 2021, 7: eabe8739

21 Zhao F, Zhou X, Shi Y, et al. Highly efficient solar vapour generation via hierarchically nanostructured gels. Nat Nanotech, 2018, 13: 489-495

22 Wei J, Wei G, Wang Z, et al. Enhanced solar-driven-heating and tough hydrogel electrolyte by photothermal effect and Hofmeister effect. Small, 2020, 16: 2004091

23 Shi Y, Ilic O, Atwater HA, et al. All-day fresh water harvesting by microstructured hydrogel membranes. Nat Commun, 2021, 12: 2797

24 Le XX, Lu W, He J, et al. Ionoprinting controlled information storage of fluorescent hydrogel for hierarchical and multi-dimensional decryption. Sci China Mater, 2019, 62: 831-839

25 Morsali M, Khan MTA, Ashirov R, et al. Mechanical control of periodic precipitation in stretchable gels to retrieve information on elastic deformation and for the complex patterning of matter. Adv Mater, 2020, 32: 1905779

26 Le X, Shang H, Yan H, et al. A urease-containing fluorescent hydrogel for transient information storage. Angew Chem Int Ed, 2021, 60: 36403646

27 Capadona JR, Shanmuganathan K, Tyler DJ, et al. Stimuli-responsive polymer nanocomposites inspired by the sea cucumber dermis. Science, 2008, 319: 1370-1374

28 Houck HA, Blasco E, Du Prez FE, et al. Light-stabilized dynamic materials. J Am Chem Soc, 2019, 141: 12329-12337

29 Chen H, Zhang J, Yu W, et al. Control viscoelasticity of polymer networks with crosslinks of superposed fast and slow dynamics. Angew Chem Int Ed, 2021, 60: 22588

30 Gao H, Zhao Z, Cai Y, et al. Adaptive and freeze-tolerant heteronetwork organohydrogels with enhanced mechanical stability over a wide temperature range. Nat Commun, 2017, 8: 15911

31 Guo H, Nakajima T, Hourdet D, et al. Hydrophobic hydrogels with fruit-like structure and functions. Adv Mater, 2019, 31: 1900702

$32 \mathrm{Xu} \mathrm{L}$, Gao S, Guo Q, et al. A solvent-exchange strategy to regulate noncovalent interactions for strong and antiswelling hydrogels. Adv Mater, 2020, 32: 2004579

33 Zhao D, Zhu Y, Cheng W, et al. A dynamic gel with reversible and tunable topological networks and performances. Matter, 2020, 2: 390403

34 Chen F, Zhou D, Wang J, et al. Rational fabrication of anti-freezing, non-drying tough organohydrogels by one-pot solvent displacement. Angew Chem Int Ed, 2018, 57: 6568-6571

35 Nian Q, Wang J, Liu S, et al. Aqueous batteries operated at $-50^{\circ} \mathrm{C}$ Angew Chem Int Ed, 2019, 58: 16994-16999

36 Ye Y, Zhang Y, Chen Y, et al. Cellulose nanofibrils enhanced, strong, stretchable, freezing-tolerant ionic conductive organohydrogel for multi-functional sensors. Adv Funct Mater, 2020, 30: 2003430

Acknowledgements This work was supported by the National Basic Research Program (2017YFC1103300) and the National Natural Science Foundation of China (51773209).

Author contributions Xu L and Qiu D conceived the experiments; Xu L performed the experiments; Xu L and Qiu D undertook the data analysis; Xu $\mathrm{L}$ and Qiu D wrote the manuscript.

Conflict of interest The authors declare that they have no conflict of interest.

Supplementary information Experimental details and supporting data are available in the online version of the paper. 


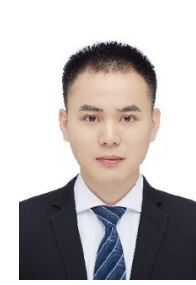

Liju $\mathbf{X u}$ is a postdoctor at the Institute of Chemistry, Chinese Academy of Sciences (ICCAS). He obtained his $\mathrm{PhD}$ degree from ICCAS in 2020. His current research focuses on the design of strong and tough polymeric gel materials and their biomedical application.

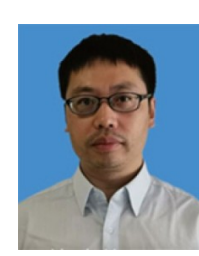

Dong Qiu is a professor at ICCAS. He obtained his BSc degree from Beijing Normal University (1999), MSc degree from ICCAS (2002) and PhD degree from the University of Bristol (2005). His current research includes the development of pH-neutral bioactive glasses, their composites with polymeric or ceramic materials and their biomedical applications.

\section{通过溶剂置换可逆切换高分子凝胶的结构与性能}

徐礼桔 ${ }^{1}$, 邱东 $1,2^{*}$

摘要 动态自适应高分子凝胶具备可逆转变的特性, 如可切换的力学 状态, 因而具有更多样化的功能及应用, 受到广泛关注. 然而, 大多数 现有材料的可切换状态或可逆转变能力有限. 本文利用高分子-高分子 间相互作用与高分子-溶剂间相互作用的竞争，发展了一种溶剂置换策 略来可逆切换动态凝胶的交联网络结构及其力学性能. 当高分子-高分 子间相互作用优于高分子-溶剂间相互作用时, 高分子网络由松散的交 联模式转变为密集的交联模式, 此时, 凝胶从软弱的状态转变为强韧的 状态. 这种转变可以通过恢复高分子-溶剂间相互作用而逆转. 此外, 该 力学性能可调的高分子凝胶在极端条件下还表现出不干燥和抗冻的性 质. 这项工作提出了一种简单而高效的可逆调控高分子凝胶结构与性 能的策略, 并为设计开发智能和适应性凝胶材料提供了理论基础. 